\title{
Articulatory phonetics of coronal stops in monolingual and simultaneous bilingual speakers of Canadian French and English
}

Francois-Xavier Brajot, Fatemeh Mollaei, Megan Callahan, Denise Klein, Shari R. Baum, and Vincent L. Gracco

Citation: Proc. Mtgs. Acoust. 19, 060064 (2013); doi: 10.1121/1.4799468

View online: https://doi.org/10.1121/1.4799468

View Table of Contents: https://asa.scitation.org/toc/pma/19/1

Published by the Acoustical Society of America

\section{ARTICLES YOU MAY BE INTERESTED IN}

Sensorimotor adaptation affects perceptual compensation for coarticulation

The Journal of the Acoustical Society of America 141, 2693 (2017); https://doi.org/10.1121/1.4979791

Modulation of auditory-motor learning in response to formant perturbation as a function of delayed auditory feedback

The Journal of the Acoustical Society of America 141, 2758 (2017); https://doi.org/10.1121/1.4981139

Toward a consensus on symbolic notation of harmonics, resonances, and formants in vocalization

The Journal of the Acoustical Society of America 137, 3005 (2015); https://doi.org/10.1121/1.4919349

Voicing-dependent vowel duration in English and French: Monolingual and bilingual production

The Journal of the Acoustical Society of America 71, 173 (1982); https://doi.org/10.1121/1.387344

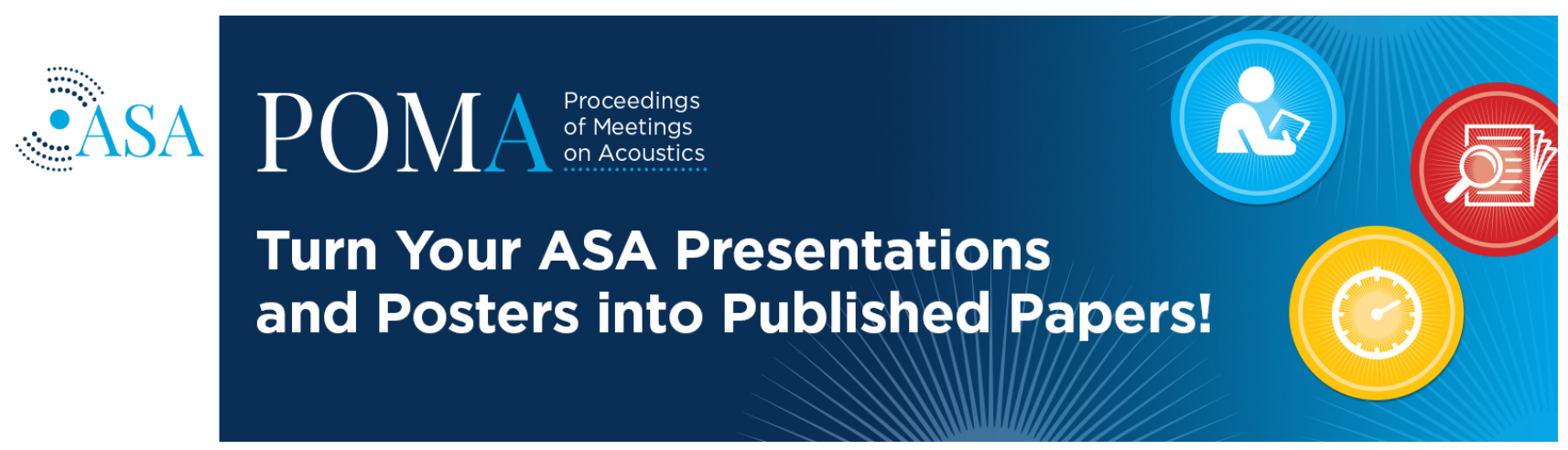




\title{
Proceedings of Meetings on Acoustics
}
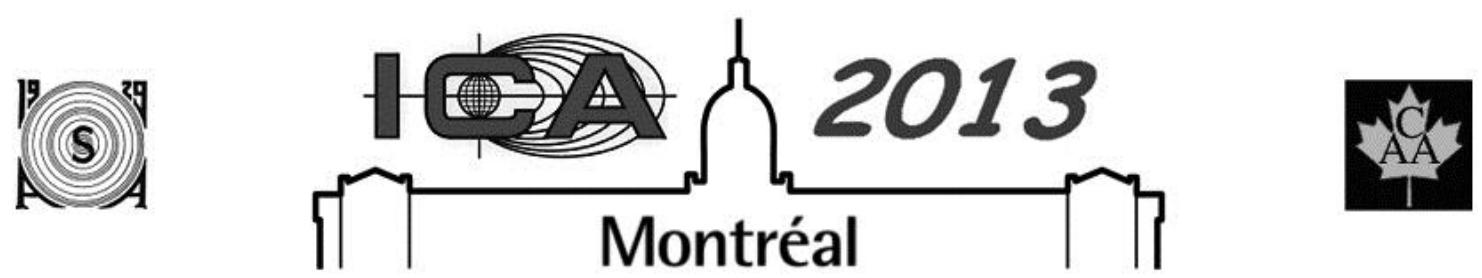

\author{
ICA 2013 Montreal \\ Montreal, Canada
}

2 - 7 June 2013

Speech Communication

Session 2aSC: Linking Perception and Production (Poster Session)

\section{2aSC20. Articulatory phonetics of coronal stops in monolingual and simultaneous bilingual speakers of Canadian French and English}

Francois-Xavier Brajot*, Fatemeh Mollaei, Megan Callahan, Denise Klein, Shari R. Baum and Vincent L. Gracco

*Corresponding author's address: School of Communication Sciences and Disorders, McGill University, 1266 Pine Avenue West, Montreal, H3G 1A8, QC, Canada, fx.brajot@mail.mcgill.ca

Previous studies of bilingual speech production have relied on individuals whose age of acquisition of their second language varies. In the proposed research, we take advantage of the unique multilingual environment of Montreal and examine speech production in individuals who have acquired two languages from birth and compare the results to monolingual speakers. Electromagnetic recordings of single-word productions were carried out on three groups of female Canadian speakers (French monolingual, English monolingual, French-English simultaneous bilingual). Spectral moment and formant transition analyses of coronal burst segments showed cross-linguistic differences across vowel contexts. Tongue place of articulation and shape likewise indicated cross-linguistic differences in static articulatory positions. Kinematic analyses further identified language-specific movement patterns that helped clarify certain results from the acoustic analyses, namely that spatiotemporal characteristics of coronal articulation help enhance vocalic dimensions important to the respective language. Similar patterns were observed among the bilingual subjects, with the notable exception that acoustic and kinematic spaces narrowed considerably, resulting in reduced overlap between languages. It appears that simultaneous bilingual speakers not only follow language-appropriate articulatory and acoustic patterns, but further minimize areas of cross-linguistic convergence otherwise found among monolingual speakers.

Published by the Acoustical Society of America through the American Institute of Physics 


\section{INTRODUCTION}

A fundamental question in bilingualism research is how two separate language systems can be organized and used in a manner that allows intra-linguistic specificity as well as cross-linguistic flexibility. Much of the research on this question has focused on lexical, grammatical and semantic competence or performance phonological and phonetic aspects, despite notable improvements in recent years, remain severely understudied (Watson, 1991). When bilingual speech sound production is addressed, moreover, the analyses are generally limited to perceptual or acoustic descriptions. We therefore conducted a study on the articulatory characteristics of bilingual speech and its acoustic consequences. This paper presents a preliminary investigation of coronal stops in Canadian French (CF) and English (CE).

Coronals make up an enormously varied set of consonants that can be found throughout the world's languages (Keating, 1990) and have brought about numerous typological descriptions (Arsenault, 2008). These descriptions are complicated by disagreements as to what constitutes the blade of the tongue (the coronal lingual point of contact), the type of constriction (as it relates to tongue shape, e.g. apical vs. laminal), and so-called "secondary articulations" such as gradations of stricture or associated tongue body position (Keating, 1990; Fleming, 2003). The articulatory instantiation of a given coronal consonant has been shown, furthermore, to vary both across languages and within speakers (Dart, 1998). Recent studies on the acoustics of CF and CE coronal stops have, nonetheless, identified consistent differences between the languages, including burst intensity and spectral mean, standard deviation and kurtosis (Sundara, 2005). Cross-linguistic discrimination by both monolingual and bilingual individuals has been shown to depend on the vowel context within which the coronal stop is placed, however (Sundara \& Polka, 2008).

Fleming (2003) provides one explanation for this phenomenon by positing that tongue body position constrains or is conditioned by coronal place of articulation. Anterior (apical or laminal) coronal consonants result in a fronted tongue body, and concomitant fronting of adjacent vowels, whereas posterior (retroflex) coronal consonants are associated with a backed tongue body and vowel retraction. Fleming's proposed typology helps explain the intra-speaker variability noted above, as well as possible vowel context effects on coronal burst acoustics and perception. Grouping apical and laminal types of constrictions under the anterior feature constraint fails to clarify the reason for the apical/laminal distinction in CF vs. CE described by Sundara (2005), however.

Arsenault (2008) suggests a three-dimensional feature classification for coronal sounds that alleviates the need to specify multiple features dependent on place of articulation. Tongue "curl" associates a convex tongue shape with the laminal feature and a concave shape with the apical feature. This feature is combined with tongue body height and "thrust" (advancement) to encompass the various coronal articulatory descriptions. An apical alveolar [t], for instance, is described as [concave] + [back], a lamino-dental [t] as [convex] $+[$ low], and a lamino-alveolar (palatal) [t] as [convex] $+[$ high]. By taking into consideration tongue body shape, in other words, variations in coronal consonant production can be specified succinctly with two or three features that incorporate both place of constriction and tongue body positioning. The result can be counter-intuitive, such as the consequence of tongue body fronting, when convex, resulting in a posterior place of articulation.

As a starting point in our analysis, we will summarize results of acoustic measures that successfully replicate findings by Sundara and colleagues (Sundara, 2005; Sundara et al., 2006) discriminating CF and CE coronal stop production. We follow up with a description of lingual position and shape in an attempt to incorporate notions forwarded by Arsenault (2008) and to determine whether the inclusion and specification of tongue body configuration aid in understanding the articulatory underpinnings for differences observed in the acoustic output. We also describe results of a kinematic assessment of lingual movement completed over the course of the release of the coronal stop moving into the adjacent vowel. Lastly, we speculate on the possible mechanism behind observed articulatory and acoustic differences between CF and CE.

\section{METHODS}

\section{Subjects}

18 female subjects were recruited for this study, divided into three experimental groups: 6 monolingual English speakers (mean 24.2 years), 6 monolingual French speakers (mean 23.8 years), and 6 simultaneous bilingual French-English speakers (mean 24.8 years). All subjects reported normal hearing and had no history of speech or language problems. 


\section{Speech sample}

The list of analyzed words is presented in the Appendix. These were selected from a larger sample taken as part of a multi-center research project on bilingual speech and language.

\section{Procedure}

All kinematic data were recorded while subjects were seated in a Karstens AG500 electromagnetic articulograph (EMA). Reference coils were placed on subject mastoids, bridge of the nose and upper incisors (at the base of the upper labial frenum). Midsagittal tongue coils were placed at $0.5,3$ and $5 \mathrm{~cm}$ from the tongue tip. Coils were also placed between the lower incisors and on the upper and lower lip. Amplitude signals for each coil were digitized at $200 \mathrm{~Hz}$. Subjects were asked to read aloud stimuli presented on video monitor placed $2 \mathrm{~m}$. in front of them. Audio was recorded with a Sennheiser microphone, with a $16 \mathrm{kHz}$ sampling rate, and synchronized with the kinematic data.

\section{Data processing}

After experimental recording, articulographic amplitude data were converted to "position" values and normalized relative to head movement. Each recorded production was segmented manually using perceptual and acoustic information. Since acoustic information for word-initial voiceless plosives begins only at burst release, all such phonemes were marked at burst onset. For consistency, voiced plosives were also marked at burst onset. Prevoicing was later determined as described in the following section.

\section{Acoustic analysis}

Formant analysis was completed using the Praat "to Burg" algorithm (Boersma \& Weenik, 2012). Individual productions were grouped according to vowel context and averaged across subjects. Vowel mid-point values were used for establishing the F1-F2 space. No attempt was made at this point to determine whether this corresponded to the vowel's steady state. Coronal plosive burst duration was determined from the marker of the initial phoneme (placed at the point of release) to that of the following vowel. Voice onset time was then determined using a semi-automatic segmentation routine that identified voice onset from the signal's short-term root-meansquare departure from baseline. Manual segmentation was required when the pre-vocalization baseline was unstable. Spectral moment analyses included the spectral mean, skewness and kurtosis of the coronal burst (from frication onset to vowel onset) at $6 \mathrm{~ms}$ increments. These values were then averaged across the entire burst duration, then across subjects, for a given vowel context.

\section{Kinematic analysis}

All kinematic signals of interest were first smoothed using a 5-point moving average. The point of maximal constriction at the anterior tongue sensor was determined from the point of minimal tangential velocity closest to the acoustic marker specified for the phoneme onset. Frontal (Anterior-Posterior) and transverse (Superior-Inferior) points at maximal constriction were then normalized by subtracting the respective axes of the upper incisor sensor. The same point of maximal constriction was used to extract position values for tongue blade (TB) and dorsum (TD) coils. A quadratic fit was then applied to the three tongue markers. This provided three quantitative coefficients of tongue shape:

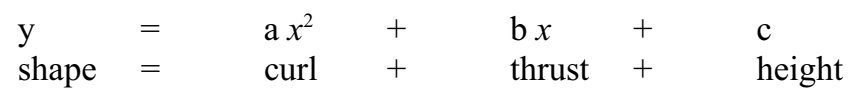

The tongue dorsum coil for one French bilingual subject was corrupt throughout the data collection; those data were therefore not included in this portion of the analysis. First and second derivatives of frontal and transverse coil positions were also calculated to determine lingual velocity and acceleration over the course of the coronal burst release segment. 


\section{RESULTS}

\section{Acoustics}

Language-specific differences were readily identified from comparison of acoustic characteristics. High vowels were produced with greater F2 (suggesting a more anterior tongue position) in English than in French, consistent with what has been reported in the literature (Escudero and Polka, 2003). Figure 1 displays the F1-F2 vowel space for target phonemes averaged across all productions. As concerns voice-onset time (VOT; see Figure 2), monolingual English plosive VOT was consistently positive; voiced and voiceless plosives could be differentiated by VOT lag. The monolingual French voiced-voiceless contrast was identified as a prevoicing/voicing lag difference. Spectral moment analysis of coronal plosive burst segments revealed greater overlap between languages, with the exception of $/ \mathrm{i} /$, which had significantly higher mean $(\mathrm{F}(1,10)=4.9, \mathrm{p}=0.05)$ and negative skew $(\mathrm{F}(1,10)=6.15, \mathrm{p}=0.03)$ in French than in English. Figure 3 shows mean and skew for word-initial /t/ bursts; values for /d/ were roughly similar. From these parameters alone, it appears that tongue backing and lip rounding both reduce the mean frequency and increase skew, leading to overlapping distributions across back vowels as can be observed for French /u/ and /a/. Kurtosis helps clarify the height distinction, at least for monolingual controls (not shown here). Lastly, formant transition analyses for English /d/ are consistent with previously reported descriptions (Steven, 2000). The slight rise in F2 for /i/ is associated with a drop of the tongue blade and minimal tongue body movement, whereas the decrease in F2 for back vowels is associated with backward movement of the tongue body. French monolingual high vowels in our data are characterized by fairly stable formants throughout the burst duration, with the possible exception of a slight rise in F1 for /y/. The greatest crosslinguistic distinction is observed in the steeper falling slope of the French / $\mathrm{u} /$, suggesting a stronger backward movement of the tongue body than in English.

Bilingual speakers followed largely similar patterns observed among monolinguals, with the exception that measured parameters shifted or were restricted in a way that reduced overlap across languages. With respect to vowels studied, for example, F2 values for English /u/ showed significantly less overlap with French /y/. Bilingual participants often produced English stop consonants with a voicing lead, unlike their monolingual English counterparts, yet with reduced ranges compared to their own and control French productions. These findings are strikingly similar to results reported by Sundara et al. (2006) on simultaneous bilingual VOT measures. As in monolingual speakers, no cross-linguistic differences in spectral moments were found among bilingual participants, except for $/ \mathrm{i} /$ spectral mean $(\mathrm{F}(1,10)=12.2, \mathrm{p}=0.006)$ and skew $(\mathrm{F}(1,10)=13.42, \mathrm{p}=0.004)$. Formant transitions for bilinguals were also very similar to their monolingual counterparts. Note however the stronger drop in F2 for English /u/, which eventually reaches values in the lower end of the monolingual /u/ distribution, overlapping the French /u/ space (cf. Figure 1).
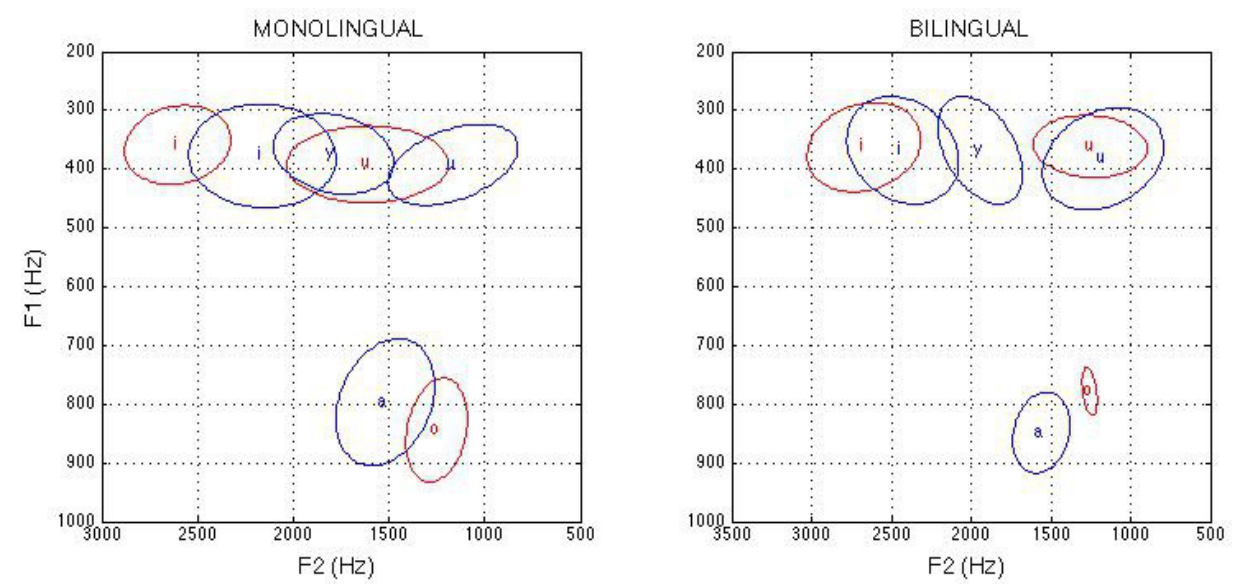

FIGURE 1. F1-F2 vowel space for target phonemes averaged across language; French is in blue. English is in red. Ellipses represent a one standard deviation phone dispersion. Values for monolingual speakers are shown in panel 1; those for bilingual speakers in panel 2. 

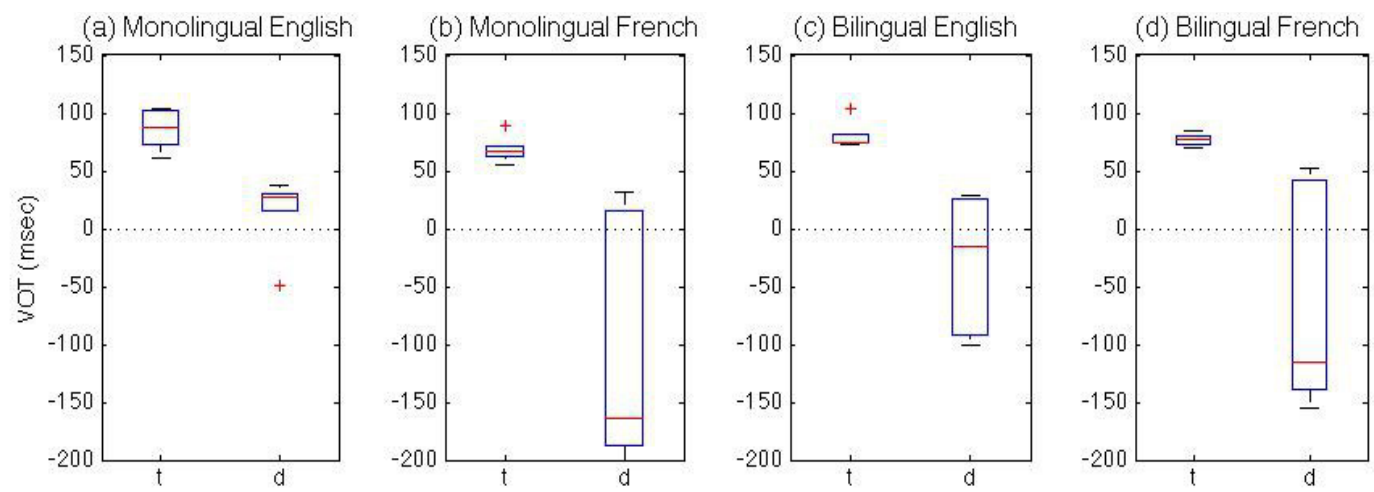

FIGURE 2. Group data on dental-alveolar voice-onset time (in $\mathrm{msec}$ ). Boxes delimit the $25^{\text {th }}$ and $75^{\text {th }}$ percentiles, with a center mark for the median. Whiskers extend to 2.7 standard deviations; points outside this range are plotted as crosses.

it/ SPECTRAL MEAN

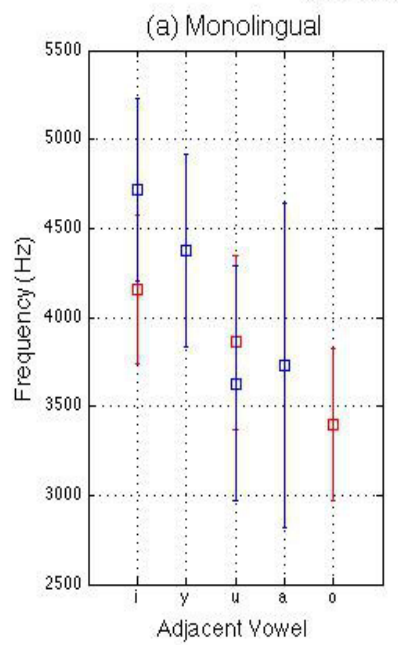

(b) Bilingual

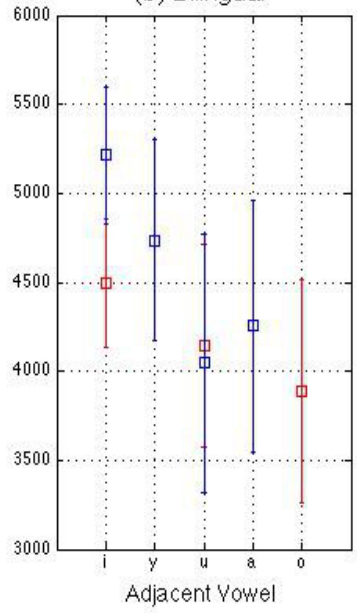

it/ SPECTRAL SKEW

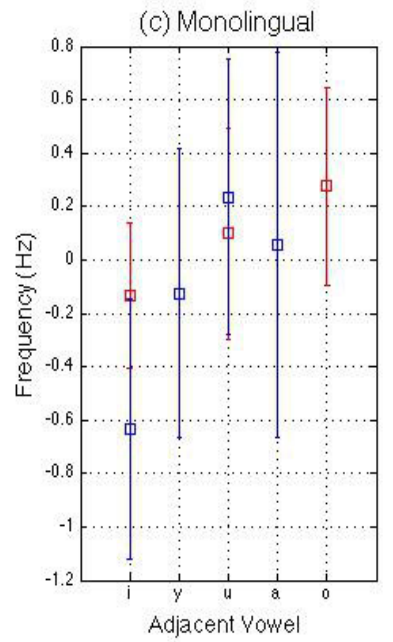

(d) Bilingual

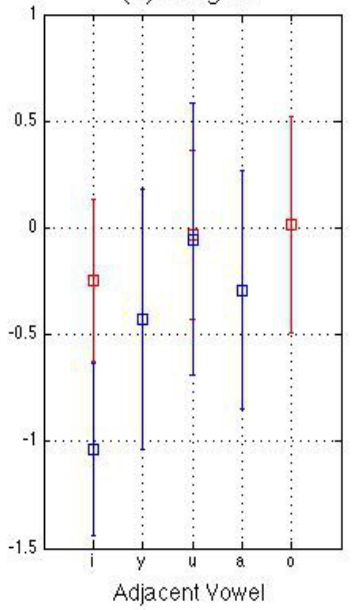

FIGURE 3. First (mean) and third (skew) spectral moments of word-initial /t/ burst segment averaged across speakers of a given group. Error bars extend to 2.7 standard deviations. Results for French productions are in blue, those for English in red.

(a) Monolingual English

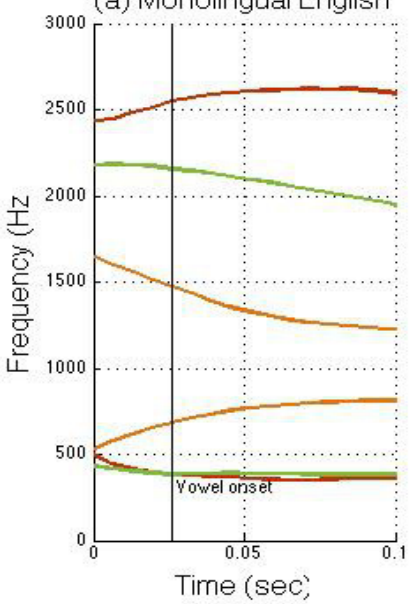

(b) Monolingual French

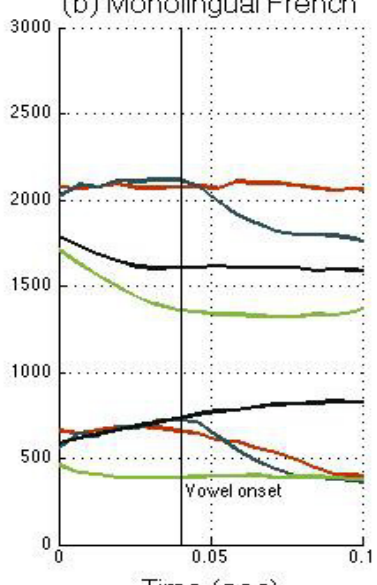

(c) Bilingual English

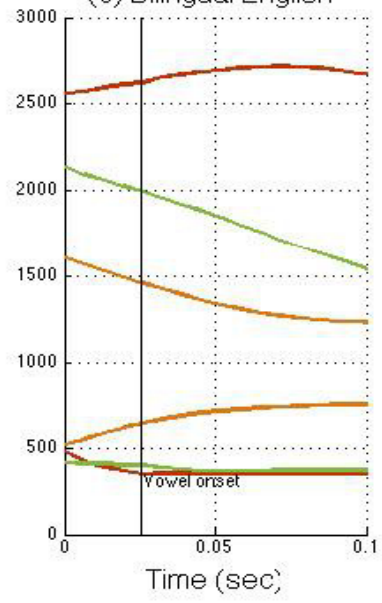

(d) Bilingual French

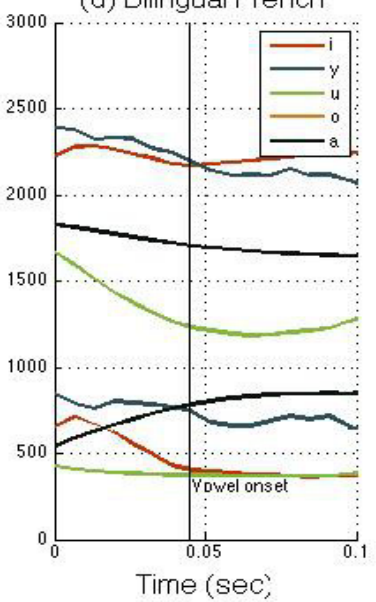

FIGURE 4. First and second format transitions from burst onset. $100 \mathrm{~ms}$ are plotted, including vowel onset identified from the acoustic output. Plotted colors represent the following vowel contexts: /i/ red, /y/ blue, /u/ green, /o/ orange, /a/ black. 


\section{Kinematics}

Cross-linguistic differences were also observed on static and dynamic measures of lingual markers. Spatial dispersion of the anterior tongue marker at the point of maximal constriction is plotted in Figure 5. A clear separation can be identified among monolingual speakers, with French coronals produced with a more anterior and inferior placement than in English (MANOVA F $(1,21)=34.12, \mathrm{p}<0.0001$ ). Quadratic fits to the three tongue markers provided measures of tongue curl (degree of convexity), height and thrust (backness). These are summarized in Figure 6. Graphically, the data suggest that monolingual English speakers articulated /t/ and /d/ with a slightly more convex and anterior tongue position. Monolingual French speakers, by contrast, show a distribution more centered around the origin (neutral curl and thrust). An anterior, convex tongue shape with a posterior, superior tongue tip position is consistent with an apical classification, commonly associated with English coronal stops (Stevens, 2000; Dart, 1998). The fairly neutral shape and more anterior tongue tip position seen for French productions is more consistent with a laminal or apicolaminal constriction (Dart, 1998).

Interestingly, static measures of position and shape were more variable among bilingual speakers. Tongue tip position at maximal constriction is slightly more anterior and inferior in French than in English, but the two distributions overlap much more than seen among monolingual subjects (MANOVA F $(1,22)=19.14, p<0.0001$ ). Indices of tongue shape likewise indicate important overlap between languages, precluding the measure for reliable discrimination between languages. Analysis of position derivatives over the course of the release segment do, however, show patterns in line with monolingual counterparts, as well as a shift in distributions that reduce crosslinguistic overlap.
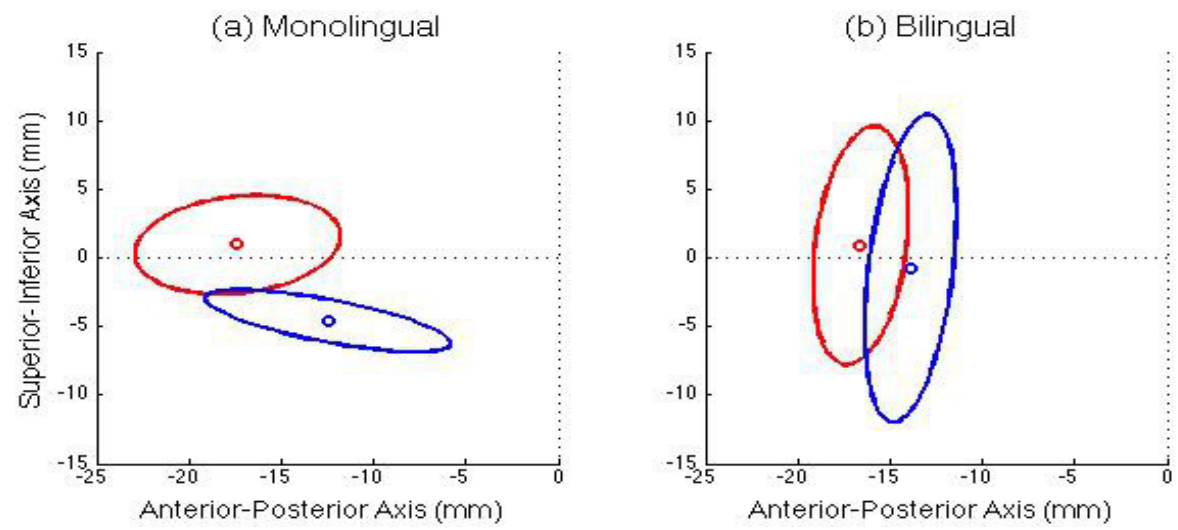

FIGURE 5. Tongue tip marker placement at the point of maximal constriction before the release of the coronal plosive. Ellipses represent 2.7 standard deviations of marker position posterior to the superior incisors, averaged across all $/ \mathrm{t} /$ and $/ \mathrm{d} / \mathrm{phonemes}$ for each speaker. French is in blue, English in red.

(a) Monolingual

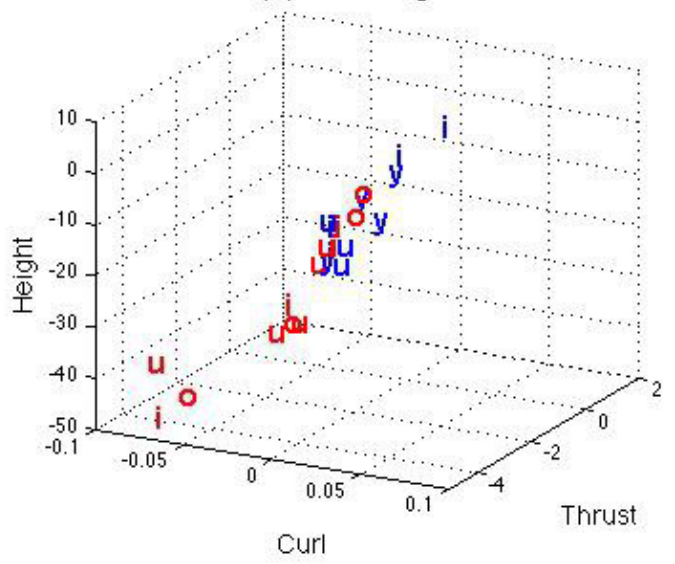

(b) Bilingual

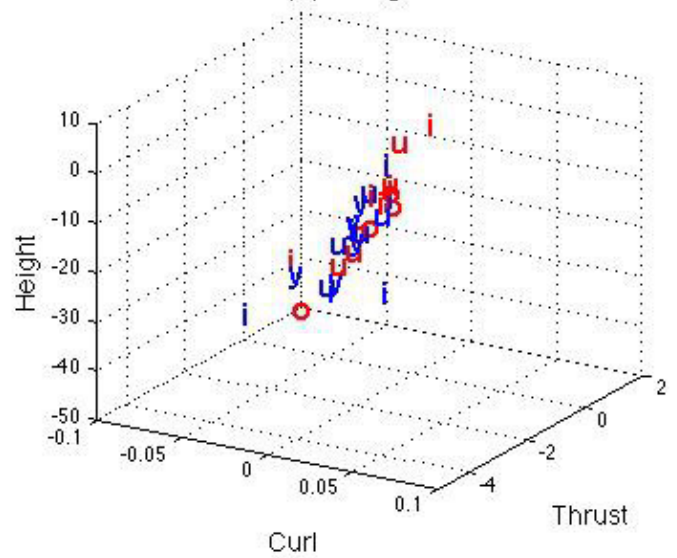

FIGURE 6. Lingual curl (negative values denote greater convexity), thrust (negative values denote more advanced position), and height during maximal constriction of /t/, averaged across subjects. French is in blue, English in red. 
Velocity and acceleration of the anterior tongue marker calculated across the release segment revealed patterns similar to those observed in the spectral moment and formant transition analyses, namely greater overlap in height categories (/i, u/ vs. /o/) among English monolinguals, as opposed to greater overlap in front-back dimensions (/i, y/ vs. /u, a/) for French monolinguals. Figure 7 displays frontal (A-P) and transverse (S-I) velocity-acceleration plots of tongue tip movement from the point of maximal constriction for $/ \mathrm{t} /$ to vowel onset. Only mean values for the monolingual groups are shown; results are essentially identical for bilingual groups. Similar patterns were also observed for the other tongue markers ('blade' and 'dorsum'). Note in particular the overlapping /i/ and /u/ curves, in both axes, for English speakers. There is relatively little movement in the A-P axis (/ti/ in fact often has positive velocity, indicating forward movement from the point of maximal constriction), but clearer S-I movement throughout the burst. Tongue tip release for French speakers in the / $\mathrm{u} /$ context reveals greater A-P negative velocity and acceleration, indicating larger backward movement, distinct from the similar patterns seen for movement in /i/ and $/ y /$ contexts. Figure 8 shows subject-averaged velocity and acceleration of tongue tip movement during the coronal release, separated by vowel context. Again, note the overlap between /i/ and /u/ contexts for English speakers, and that between /i/ and /y/ versus /u/ and /a/ for French speakers. Results from bilingual subjects are also plotted to show the reduced dispersion and clearer segregation across languages.

(a) Monolingual English

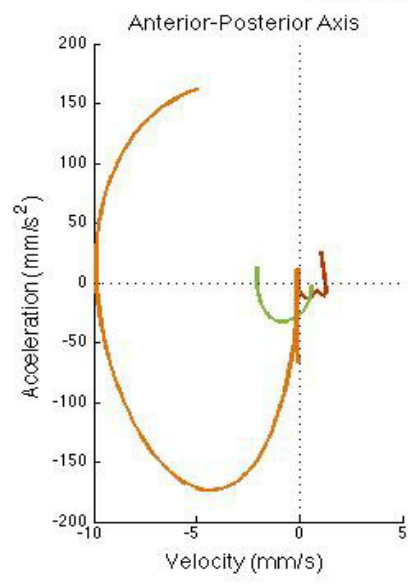

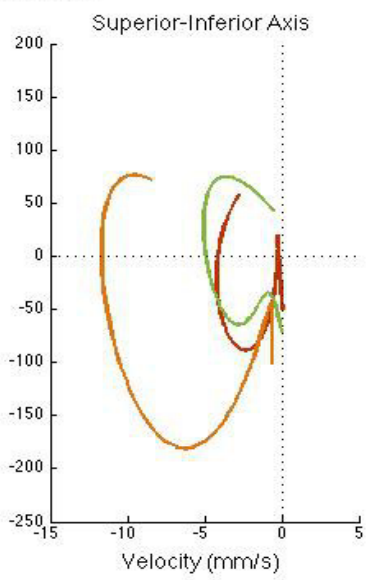

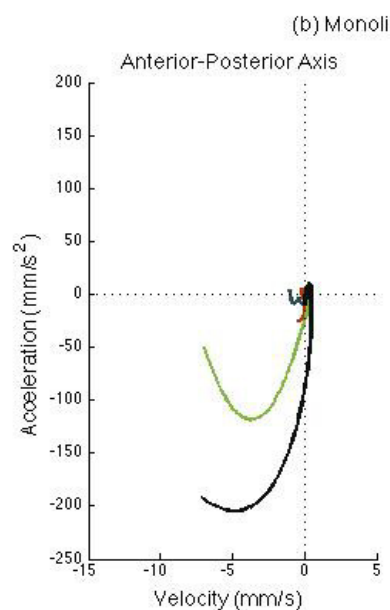

b) Monolingual French

FIGURE 7. Velocity-acceleration plots of lingual apical movement during the coronal burst release separated by vowel context and averaged across subjects in a given language category. Panels 1-2 are the A-P and S-I axes for monolingual English subjects' productions, respectively; Panels 3-4 are the A-P and S-I axes for monolingual French subjects' productions. Plotted colors represent the following vowel contexts: /i/ red, /y/ blue, /u/ green, /o/ orange, /a/ black.
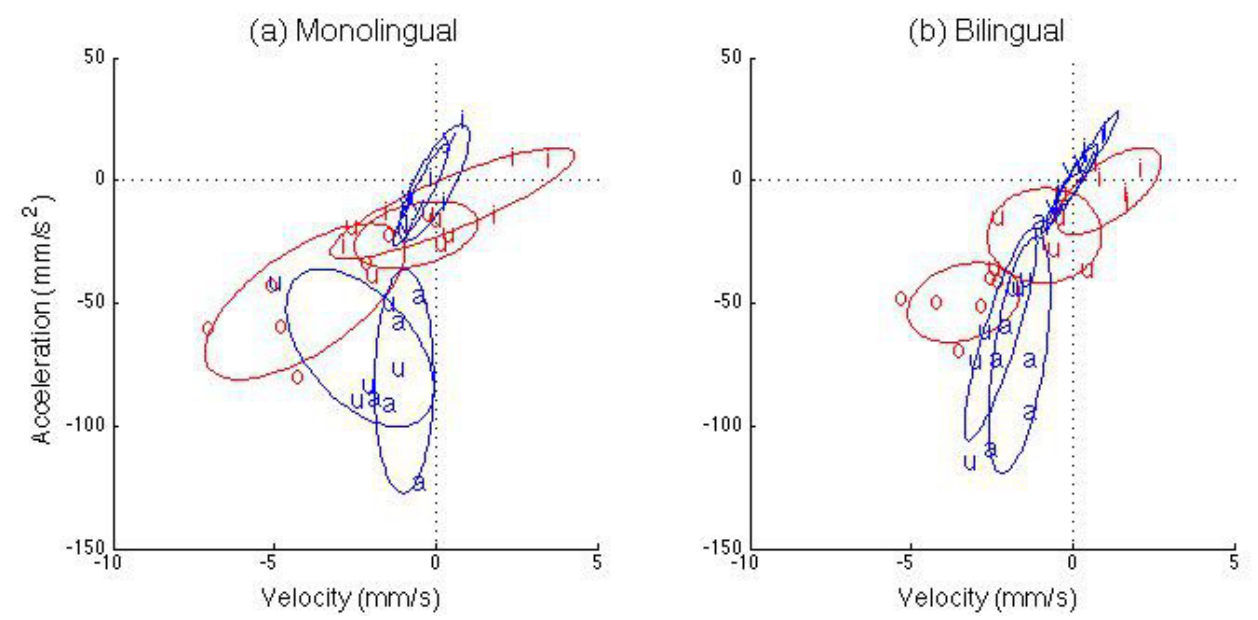

FIGURE 8. Mean velocity and acceleration of lingual apical movement during the coronal burst release separated by vowel context and averaged across subjects in a given language category. Values for monolingual speakers are shown in panel 1; those for bilingual speakers in panel 2. French is in blue, English in red. 
To summarize, in line with previous research on the issue of coronal articulatory and acoustic phonetics (e.g. Dart, 1998), articulation of English coronal stops tend to be apical, whereas in French they tend to be laminal or distributed, despite the fact that the place or type of constriction is not phonemic in either language. The fact that bilingual speakers follow similar kinematic and acoustic patterns while reducing cross-linguistic overlap supports the notion that these are important characteristics to each language. When measured relative to vowel context, both acoustic and kinematic measures reveal greater similarities in front-back dimensions for English, versus high-low dimensions for French. From a typological standpoint, this may be argued to result from vocalic dimensions of importance in the respective languages. English, with a more complex array of high-low categories, may allow for more overlap within a height category and across the thrust dimension. French categories, when limited to F1-F2 space, show greater complexity along the front-back dimension (ignoring, for now, the importance of F3 and F2' related to the rounding distinction). Overlap is therefore expected within a thrust category and across height. Differences in coronal consonant tongue shape and placement between languages, like French and English, that do not phonemically distinguish apicality may, in other words, result from a more global or contextual requirement to emphasize vocalic dimensions relevant to the given language. From an articulatory standpoint, a convex tongue body and apical coronal gesture may facilitate subsequent height distinctions, whereas a distributed tongue shape and laminal coronal gesture arise in languages that emphasize vocalic front-back distinctions.

Considering the limited database with which we are working here, this interpretation remains very speculative. The strongest argument for this hypothesis is the overlap between acoustic and articulatory patterns for coronal release before $/ \mathrm{u} /$. Acoustic measures of the vowel are very similar across languages, with the notable exception that second formant values in English spread into French /y/ F2 values (greater variability in the "thrust" dimension). Were the French / $/$ / articulated like the English / $t /$, the effect of rounding in the /y/ context would result in a drop in spectral moment values, effectively overlapping the range of values seen for /tu/.

The lack of clear language-specific tongue configurations among simultaneous bilinguals casts some doubts on this hypothesis. Bilingual speakers clearly follow both articulatory and acoustic patterns observed among bilinguals. The finding that tongue shapes are undifferentiated in the bilingual group may suggest that the measure is not essential for the production of the different coronal release characteristics. Variability in lingual configuration, in other words, provides the flexibility needed to transition between languages. It is also possible that the measure of tongue shape is not effective at capturing configurations important to coronal articulation. Adding a fourth marker at the actual tongue tip, for instance, and modeling the tongue with a cubic polynomial or spline may more positively identify configurations such as a convex body with a dipping blade and raised apical tongue tip.

\section{CONCLUSION}

Acoustic and kinematic analyses were conducted on simultaneous bilingual and monolingual control individuals' productions of word-initial coronal stops in single-syllable words. Results of acoustic measures including vowel formant analysis and consonantal voice-onset time, spectral moments and formant transitions demonstrated clear cross-linguistic differences in both monolingual and bilingual participants. The ranges of observed values were more limited among simultaneous bilingual speakers, moreover, reducing areas of crosslinguistic overlap identified among monolinguals. A similar phenomenon was seen in velocity-acceleration profiles of tongue movement during the coronal burst segment. Static articulatory measures of tongue tip position and lingual shape during maximal constriction of the coronal stop consonant were associated with increased variability among bilingual subjects, however, while monolingual subjects maintained clear cross-linguistic differences.

The retention of language-specific patterns in dynamic articulatory measures among simultaneous bilingual speakers, despite concomitant changes that reduced cross-linguistic overlap, hints at an important mechanism relevant to the production of coronal stops in these languages. Similarities in both kinematic and acoustic parameters dependent on vowel context suggest that certain lingual configurations may function to enhance thrust or height distinctions relevant to a given language's vowel inventory. The more forward placement and distributed shape of the coronal stops in French may heighten front-back distinctions as the articulation moves toward the following vowel. The posterior placement and convex tongue shape produced by English speakers, on the other hand, may result in stronger displacements along the height dimension. The lack of clear lingual configurations among the bilingual speakers indicates that tongue shape is not necessary to maintain language appropriate acoustic characteristics, but may be an aspect bilinguals exploit to permit inter-linguistic flexibility. Too few vowel contexts were included in this analysis to make any strong conclusions, however. Similar analyses in different vowel contexts are necessary to determine the validity of these claims. 


\section{ACKNOWLEDGMENTS}

We would like to thank Mark Tiede for providing an extensive library of Matlab functions for the acquisition and processing of articulographic data. We would also like to thank Doug Shiller for assistance in technical issues related to the AG500 articulograph. Funding was provided by a grant from the Social Sciences and Humanities Research Council (SSHRC).

\section{APPENDIX}

TABLE 1. List of selected tokens.

\section{English}

French

\begin{tabular}{ccccccc}
$/ \mathbf{i} /$ & $/ \mathbf{u} /$ & $/ \mathbf{o} /$ & $/ \mathbf{i} /$ & $/ \mathbf{u} /$ & $/ \mathbf{a} /$ & $/ \mathbf{y} /$ \\
\hline tea & two & top & titre & toux & tas & tue \\
team & tool & tock & tigre & tousse & tape & tube \\
teach & tune & tot & tire & touche & tache & tuque \\
deem & do & doll & dit & doux & date & dû \\
deep & doom & dock & dites & douce & dalle & duc \\
deal & dune & dot & dix & douche & dame & dure \\
\hline
\end{tabular}

\section{REFERENCES}

Arsenault, P. (2008). "On feature geometry of coronal articulations," Toronto Working Papers Ling. 29, 1-21.

Boersma, P., \& Weenik, D. (2012). "Praat: Doing phonetics by computer," http://www.praat.org. (Last viewed 01 Jan. 2013.)

Dart, S. (1998). "Comparing French and English coronal consonant articulation,” J. Phonetics 26, 71-94.

Escudero, P. \& Polka, L. (2003). "A cross-language study of vowel categorization and vowel acoustics: Canadian English versus Canadian French," in Proceedings of the 15 $5^{\text {th }}$ International Congress of Phonetic Sciences, Barcelona, August 3-9, pp. 836-864.

Fleming, E. (2003). "The relationship between coronal place and vowel backness," Phonology 20(3), 335-373.

Keating, P.A. (1990). “Coronal places of articulation,” UCLA Working Papers in Phonet. 74, 35-60.

Sundara, M. (2005). "Acoustic-phonetics of coronal stops: A cross-language study of Canadian English and Canadian French,” J. Acoust. Soc. Am. 118(2), 1026-1037.

Sundara, M. \& Polka, L. (2008). "Discrimination of coronal stops by bilingual adults: The time and nature of language interaction," Cogn. 106, 234-258.

Sundara, M., Polka, L. \& Baum, S. (2006). "Production of coronal stops by simultaneous bilingual adults," Bilingual. Lang. Cogn. 9(1), 97-114.

Stevens, K.N. (2000). Acoustic Phonetics (MIT Press, Boston).

Watson, I. (1991). "Phonological processing in two languages," in Language Processing in Bilingual Children, edited by E. Bialystok (Cambridge University Press, Cambridge), pp.25-48. 